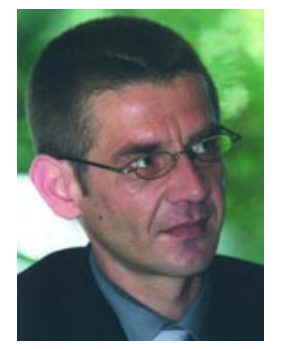

Andreas Ströhle

\title{
Experimentelle Provokation von Panikattacken
}

\author{
Andreas Ströhle \\ Klinik für Psychiatrie und Psychotherapie, Campus Charité Mitte, Berlin
}

psychoneuro 2005; 31 (3): 145-153

$\mathrm{P}$ lötzlich und unerwartet, d.h. ohne ersichtlichen äußeren Auslöser auftretende Panikattacken können wegen ihres klar abgrenzbaren Verlaufs, sowie ihres charakteristischen Musters somatischer, emotionaler, kognitiver und behaviouraler Symptome als eine prototypische Erscheinungsform von Angst angesehen werden. Panikattacken treten bei $10-30 \%$ der Bevölkerung auf und sind ein bedeutsamer Risikomarker für das spätere Auftreten psychiatrischer Erkrankungen. Darüber hinaus sind sie ein spezifischer Risikofaktor für die Entwicklung einer Panikstörung. Die Panikstörung ist durch das wiederholte Auftreten spontaner Panikattacken, ausgeprägte und persistierende Erwartungsangst und häufig auch durch ein agoraphobes Vermeidungsverhalten charakterisiert. Durch die experimentelle Provokation von Panikattacken, z.B. mittels einer Laktat-Infusion oder pharmakologischer Interventionen, ergibt sich, neben den sogenannten Modellpsychosen, die in der Psychiatrie nahezu einzigartige Möglichkeit, das zentrale klinische Symptom, beim Menschen experimentell zu erzeugen und standardisiert zu untersuchen. Im Folgenden werden die wichtigsten respiratorischen und pharmakologischen Provokationsverfahren dargestellt. Am Beispiel des

Die diagnostische Symptomprovokation hat in der Medizin eine lange Tradition und wird z.B. bei der koronaren Herzerkrankung oder dem Diabetes mellitus angewandt. In der Psychiatrie wird die experimentelle Symptomprovokation z.B. eingesetzt, um neurobiologische Grundlagen psychiatrischer Erkrankungen zu untersuchen. Plötzlich und unerwartet, d.h. ohne ersichtlichen äußeren Auslöser auftretende Panikattacken können wegen ihres klar abgrenzbaren Verlaufs, sowie ihres charakteristischen Musters somatischer, emotionaler, kognitiver und behavioraler Symptome als eine prototypische Erscheinungsform von Angst angesehen werden. Seit Pitts \& McClure 1967 erstmals beschrieben, dass eine Laktatinfusion bei Patienten mit Panikstörung panikogen wirkt, wurde die experimentelle Provokation von Panikattacken, auch mit anderen Stimuli, genutzt, um die Neurobiologie der Panikstörung zu charakterisieren. Serotonerg, noradrenerg, aber auch GABA-erg vermittelte Panikattacken verweisen auf unterschiedliche Mechanismen, die an der Entstehung von Panikattacken beteiligt sind. Neben primär pathophysiologisch orientierten wissenschaftlichen Untersuchungen kann die experimentelle Symptomprovokation bei der Panikstörung z.B. auch als Therapie i.S. einer Exposition verwendet werden. Zudem lassen sich damit mögliche neue Therapieansätze untersuchen. Dies wird hier am Beispiel des atrialen natriuretischen Peptids dargestellt. Obwohl die Neurobiologie der Angst in vielen Bereichen noch ungeklärt ist, stellt die experimentelle Provokation von Panikattacken eine Möglichkeit dar, standardisiert so komplexe Verhaltensweisen wie Angst und Panik besser zu verstehen und neue Behandlungsstrategien zu entwickeln.

atrialen natriuretischen Peptids (ANP) wird gezeigt, wie experimentell induzierte Panikattacken als Modell herangezogen werden können, um mögliche neue Therapiestrategien zu entwickeln. Möglichkeiten und Grenzen experimentell induzierter Panikattacken werden ebenso diskutiert wie Fragen der Validität.

\section{Experimentelle Provokation von Panikattacken} Allgemeine Voraussetzungen

Die Sicherheit während der experimentellen Symptomprovokation steht an erster Stelle. Da die Provokationsverfahren an sich nicht gefährlich sind, muss vor der Untersuchung ausgeschlossen werden, dass eine andere Erkrankung besteht, die durch eine experimentell induzierte Panikattacke verschlechtert werden könnte. Informed Consent und eine medizinische und psychiatrische Überwachung während der Untersuchung sind weitere Voraussetzungen für die Durchführung solcher Studien. Um die Pathophysiologie der Panikstörung zu untersuchen, sollten die entsprechenden Patienten 


\section{Tab. 1 Experimentell induzierte Panikattacken}

\begin{tabular}{|c|c|c|c|}
\hline Verfahren & Mechanismus & Atmung & Stresshormonsystem \\
\hline Laktat & $\mathrm{pH}, \mathrm{pCO}_{2}$ & $\uparrow$ & $=$ \\
\hline $\mathrm{CO}_{2}$ & $\mathrm{pCO}_{2}$ & $\uparrow$ & $=$ \\
\hline CCK-4 & CCK-B-Rezeptor & $\uparrow$ & $\boldsymbol{\uparrow}=$ \\
\hline mCPP, Fenfluramin & 5-HT-Rezeptor & $=$ & $\uparrow$ \\
\hline Yohimbin & Alpha-2-Rezeptor & $=$ & $\uparrow$ \\
\hline Inverser BZD-Agonist & GABA $_{A}$-Rezeptor & $=$ & $\uparrow$ \\
\hline
\end{tabular}

medikamentenfrei sein und an keiner anderen psychiatrischen Erkrankung leiden. Als Kontrollprobanden müssen solche mit positiver Familienanamnese für eine Panikstörung und solche mit Panikattacken in der Vorgeschichte ausgeschlossen werden. Bei den Patienten müssen Studien mit experimenteller Provokation von Panikattacken in ein verhaltenstherapeutisches Behandlungskonzept eingebunden sein. Der wissenschaftliche Erkenntnisgewinn sollte nicht durch andere Studien erreicht werden können. In Abbildung 1 ist als standardisiertes Erfassungsinstrument experimentell induzierter Panikattacken das Panikinventar gezeigt. Es handelt sich hierbei um eine Erweiterung des international weit verbreiteten API Scores (Acute Panik Inventory) (6).

\section{Provokationsverfahren}

Verschiedene panikogen wirksame Substanzen mit ihren Mechanismen und Auswirkungen auf das Stresshormonsystem und die Atmung sind in Tabelle 1 aufgeführt. Laktat und $\mathrm{CO}_{2}$ werden als respiratorische Provokationsverfahren $\mathrm{zu}^{-}$ sammengefasst und den pharmakologischen Provokationsverfahren mit jeweils klaren, Rezeptor-assoziierten Mechanismen gegenüberge-

\section{Tab. 2 Ziele experimenteller Symptom-} provokation in der Medizin

\begin{tabular}{ll} 
Wissenschaft & Klinik \\
\hline - Pathophysiologie & • Diagnostik \\
- Behandlung & - Therapieprädiktion \\
- Subgruppen & - Response-/Relapse- \\
- Genetik & prädiktion \\
& - Behandlung
\end{tabular}

stellt. Respiratorische Provokationsverfahren führen über $\mathrm{pH}$ und $\mathrm{pCO}_{2}$ Veränderungen und, vermittelt über medulläre Chemorezeptoren, zu einer Stimulation der Atmung.

Laktat: Die Infusion einer 0,5molaren Natrium-Laktat-Lösung ist die am besten untersuchte Methode zur experimentellen Provokation von Panikattacken. Bereits 1967 beschrieben Pitts und McClure (12) das Auftreten von Laktat-induzierten Panikattacken bei Patienten mit Angsterkrankungen, nicht jedoch bei gesunden Kontrollprobanden. Die Panikogenität von Laktat findet sich nicht nur bei Patienten mit einer Panikstörung, sondern auch bei Patienten, die Panikattacken in der Anamnese hatten, sowie bei Patientinnen mit prämenstruellem Syndrom. Eine antipanisch wirksame Psychopharmakotherapie mit Antidepressiva oder Benzodiazepinen blockiert Laktat-induzierte Panikattacken. Der Mechanismus, über den Laktat panikogen wirkt, ist noch nicht eindeutig geklärt. Die anfängliche Hypothese, dass Laktat über eine Hypokalzämie Panikattacken auslöst, konnte nicht bestätigt werden. Vielmehr wird diskutiert, dass die laktatbedingte metabolische Alkalose zu einer Vasokonstriktion der hirnversorgenden Gefäße führt, welche eine zerebrale Ischämie mit erhöhtem Laktat/Pyruvat-Quotienten und abfallendem $\mathrm{pH}$ in den medullären Chemorezeptoren zur Folge hat. Panikattacken würden demzufolge als Folge der Fehlwahrnehmung von Chemorezeptoren, die eine lebensbedrohliche zentrale Hypoxie und Azidose vermitteln, entstehen. Eine andere Hypothese geht ebenfalls von einer metabolischen Alkalose aus, wobei dem aus Laktat entstehenden Bikarbonat eine zentrale Rolle zugeschrieben wird. Bikarbonat wird zu Karbonsäure und in der Folge $\mathrm{zu} \mathrm{CO}_{2}$ umgewandelt. $\mathrm{CO}_{2}$ könnte eine zentrale Hyperkapnie zur Folge haben und über eine Stimulation medullärer Chemorezeptoren die Atmung beschleunigen. Da Hyperkapnie den Locus Coeruleus stimuliert, könnten Laktatinduzierte Panikattacken auch, oder zumindest teilweise zentral noradrenerg vermittelt sein.

Kohlendioxid $\left(\mathrm{CO}_{2}\right)$ : Die $\mathrm{CO}_{2}$-Inhalation ist ein ebenfalls gut charakterisiertes Modell experimentell induzierter Panikattacken. Panikattacken die durch Inhalation von 5\% $\mathrm{CO}_{2}$ induziert werden, sind gekennzeichnet durch einen Anstieg des Atemzugvolumens (7) im Sinne eines überaktiven respiratorischen Systems. Die Wirkungen von $\mathrm{CO}_{2}$ werden durch Rezeptoren in der Lunge, dem Aortenbogen, den Carotiden und der Medulla vermittelt. Darüber hinaus konnte im Tierexperiment gezeigt werden, dass $\mathrm{CO}_{2}$ dosisabhängig die Feuerrate des Locus Coeruleus erhöht. Hinweise für eine genetische Grundlage der $\mathrm{CO}_{2}$-Hypersensitivität (11) ermöglichen die Verwendung dieses biologischen Markers i.S. eines Endophänotyps auch in genetischen Untersuchungen.

Cholecystokinin Tetrapeptid (CCK-4): CCK-4 nimmt eine Sonderstellung zwischen den pharmakologischen Panikogenen wie Yohimbin und den respiratorischen panikogenen Laktat und $\mathrm{CO}_{2}$ ein: es stimuliert die Atmung und das Stresshormonsystem. De Montigny konnte erstmals zeigen, dass die Gabe von CCK-4 Panikattacken hervorrufen kann. Weitere Studien ergaben, dass Patienten mit einer Panikstörung eine CCK-4-Hypersensitivität haben (2). Der Versuch, durch Entwicklung spezifischer CCK-B-Rezeptorantagonisten einen neuen Therapieansatz bei Patienten mit Panikstörung zu entwickeln, war bisher jedoch nicht erfolgreich. Inwiefern dies mit der fehlenden Bioverfügbarkeit der bisheri- 
gen Substanzen zusammenhängt, ist derzeit noch offen.

In eigenen Untersuchungen waren CCK-4-induzierte Panikattacken von Patienten mit Panikstörung von einer starken Aktivierung der hypophysären ACTH-Sekretion begleitet, ohne dass es zu einer vermehrten Kortisol-Freisetzung kam (24). Auch wenn die Grundlagen für diese Entkoppelung im Stresshormonsystem noch unklar sind, verdeutlicht der fehlende Anstieg der Kortisol-Konzentrationen bei gleichzeitiger ACTH-Stimulation bei Patienten mit CCK-4-induzierten Panikattacken, dass Panikattacken von Patienten mit Panikstörung ohne vermehrte Kortisol-Sekretion mit einer zentralen Aktivierung des HPA-Systems und vermehrter CRH-Freisetzung einhergehen könnten. Dies wird durch die Beobachtung gestützt, dass bei Patienten mit spontanen oder situativ getriggerten Panikattacken ebenfalls kein Kortisolanstieg nachweisbar war.

Noradrenerge und serotonerge Neurotransmission: Die durch serotonerge oder noradrenerge Stimulation erzeugte Angstsymptomatik ist nur eingeschränkt mit der von einer Hyperventilation begleiteten Laktat-induzierten oder spontanen Panikattacke vergleichbar. Die erzeugte klinische Symptomatik wurde daher eher mit der Erwartungsangst oder Symptomen der generalisierten Angststörung in Verbindung gebracht. Auch findet sich im Gegensatz zu spontanen und Laktat-induzierten Panikattacken bei den sogenannten pharmakologischen Panikogenen eine Aktivierung des HPA-Systems (Tab. 1).

Yohimbin hat den am besten charakterisierten Wirkmechanismus experimentell induzierter Angst. Dieser $\alpha_{2}$-Adrenorezeptor-Antagonist erhöht die noradrenerge Aktivität durch Blockade der noradrenergen Autorezeptoren am Locus Coeruleus. Präklinische Studien von Redmond konnten zeigen, dass die elektrische Stimulation des Locus Coeruleus zu panikattackenartiger Symptomatik führt. Im Vergleich zu Kontrollprobanden entwickeln Patienten mit Panikstörung nach oraler Gabe von Yo- himbin vermehrt Angst und Panikattacken (4). Hingegen hat der $\alpha_{2}$-Rezeptor-Agonist Clonidin, eine transiente antipanische Aktivität, die jedoch aufgrund von Nebenwirkungen therapeutisch nicht genutzt werden kann. Die akute Clonidin-Gabe war bei Patienten mit Panikstörung stärker anxiolytisch wirksam als bei Kontrollprobanden und führte zu einem starken Abfall der Plasma-Konzentrationen von MHPG, dem peripheren Metaboliten von Noradrenalin. Imipramin blockiert zwar spontane Panikattacken und die Aktivität des Locus Coeruleus, hat aber bemerkenswerterweise keinen Einfluss auf die Yohimbin-induzierte Symptomatik.

Etwa 70\% des im ZNS nachweisbaren Noradrenalins wird im Locus Coeruleus, einer bilateralen Ansammlung von mehreren tausend noradrenergen Nervenzellen im dorsalen Tegmentum, produziert. Der Locus Coeruleus hat u.a. efferente Verbindungen zu Hypothalamus, Hippokampus, der Amygdala und Bereichen des Kortex. Afferenzen erhält der Locus Coeruleus u.a. von hypothalamischen Kernen, wobei Projektionen von CRH produzierenden Neuronen aus dem Nucleus paraventricularis für das Verständnis der Beziehung von Locus Coeruleus-Aktivität und neuroendokriner Stressreaktion von zentraler Bedeutung sind. Die Aktivität des Locus Coeruleus wird durch $\alpha_{2}$-Adrenorezeptoren moduliert. Die Stimulation dieser Autorezeptoren senkt die Feuerrate der noradrenergen Neurone und vermindert somit die Freisetzung von Noradrenalin. Noradrenalin und auch der $\beta$-Adrenorezeptor-Agonist Isoprenalin, der paradoxerweise nicht die Blut-HirnSchranke passieren kann, wirken beim Menschen anxiogen. Imipramin und andere trizyklische Antidepressiva reduzieren die Feuerrate des Locus Coeruleus.

Eine gestörte serotonerge Neurotransmission bei der Panikstörung wurde aufgrund der guten klinischen Wirksamkeit selektiver Serotonin Reuptake-Inhibitoren (SSRI) angenommen. SSRIs sind jedoch auch bei anderen psychiatrischen Erkrankungen wie der Depression,

\section{Abb. 1 Panikinventar}

Messzeitpunkt I (MZ I): Bitte beurteilen Sie, inwiefern die nachfolgend aufgeführten Befindlichkeiten für Sie im Moment zutreffen. Wenn ja, geben Sie bitte den Schweregrad an Messzeitpunkt II-III (MZ II-III): Bitte beurteilen Sie, die maximale Symptomausprägung seit der letzen Befragung

$\begin{array}{ll}\text { überhaupt nicht } 0 \\ \text { wenig } & 1\end{array}$

stark

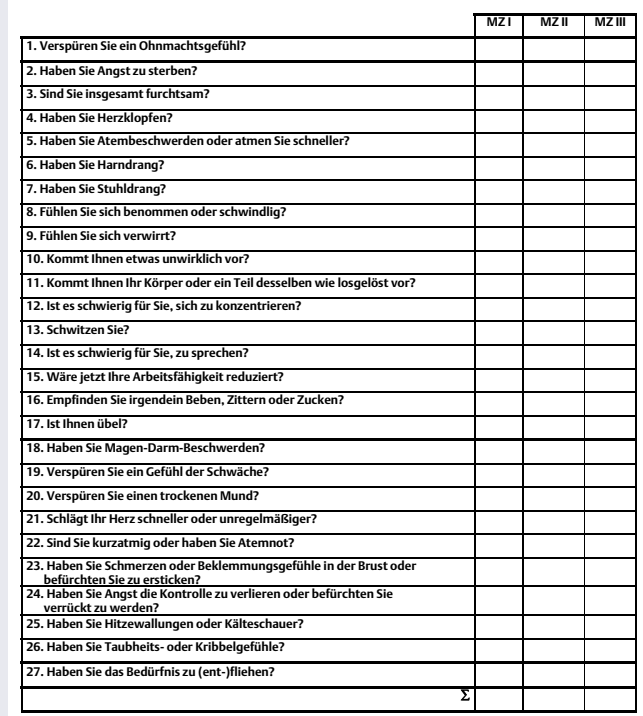

der Zwangsstörung, PTSD, bei Phobien und der generalisierten Angststörung Mittel der 1. Wahl in der Psychopharmakotherapie. Relativ spezifisch für Patienten mit Panikstörung scheint hingegen die Verstärkung der Angstsymptomatik bei $\mathrm{zu}$ rascher Aufdosierung zu sein. Dies wird auf eine Hypersensitivität postsynaptischer Serotonin-Rezeptoren zurückgeführt, was durch Studien mit dem Serotonin-Agonisten m-Chlorophenylpiperazin (mCPP) ebenfalls unterstützt wird. Die intravenöse Gabe von mCPP löst bei Patienten mit Panikstörung, und in höherer Dosierung auch bei gesunden Kontrollprobanden, Angst aus. Die Symptomatik konnte durch Ritanserin, einen 5- $\mathrm{HT}_{2}$-Antagonisten, blockiert werden (15). Oral verabreicht führt mCPP nur bei Patienten mit Panikstörung, nicht jedoch bei gesunden Kontrollprobanden $\mathrm{zu}$ Angst (8). Ähnliche Befunde wurden auch für Fenfluramin, eine Substanz die präsynaptisch Serotonin freisetzt, beobachtet: auch hier traten Panikattacken bei Patienten mit Panikstörung häufiger auf. Der partielle 5-HT ${ }_{1 \mathrm{~A}}$-Rezeptor Agonist Buspiron ist bei Patienten mit generali- 


\begin{tabular}{|c|c|c|c|c|}
\hline \multirow[t]{2}{*}{ Tab. 3} & \multicolumn{4}{|c|}{$\begin{array}{l}\text { Behandlungssensitivität } \\
\text { experimentell induzierter } \\
\text { Panikattacken }\end{array}$} \\
\hline & TZA & SSRI & BZD & VT \\
\hline Laktat & + & + & $+1-$ & + \\
\hline $\mathrm{CO}_{2}$ & + & + & + & $?$ \\
\hline CCK-4 & + & + & + & $?$ \\
\hline Yohimbin & - & + & + & $?$ \\
\hline
\end{tabular}

sierter Angststörung, nicht jedoch bei Patienten mit Panikstörung wirksam. Während die anxiolytische Aktivität von Buspiron mit einer präsynaptischen Hemmung serotoninerger Aktivität in Verbindung gebracht wurde, kann Buspiron in höheren Dosierungen über eine Aktivierung postsynaptischer 5-HT-Rezeptoren bei Patienten mit einer Panikstörung anxiogen wirken. Eine abgeschwächte Aktivierung des HPA-Systems und ein vermindertes Absinken der Körpertemperatur auf die Gabe von Ipsapiron bei Patienten mit Panikstörung wurde als Ausdruck subsensitiver prä- und postsynaptischer $5-\mathrm{HT}_{1 \mathrm{~A}}$-Rezeptoren an- gesehen. Da Ipsapiron weder bei Kontrollprobanden noch bei Patienten mit Panikstörung anxiogen wirkt, wird eine komplexe Dysfunktion des serotoninergen Systems angenommen.

GABA-erge Neurotransmission: Die anxiolytische Aktivität von Benzodiazepinen und die Anxiogenität von inversen Agonisten an den Benzodiazepin-GABA $A_{A}$-Rezeptoren sind gut charakterisiert. Während es bei Patienten mit Panikstörung Hinweise für eine verminderte Empfindlichkeit gegenüber den Effekten von Benzodiazepinen gibt (28), sind inverse Agonisten der Benzodiazepin-GABA $A_{A}$-Rezeptoren bei Patienten mit Panikstörung nicht untersucht. Als Benzodiazepin-RezeptorAntagonist blockiert Flumazenil die meisten pharmakologischen Effekte von Benzodiazepinen, inklusive der sedierenden, muskelrelaxierenden, anxiolytischen und antikonvulsiven. Daneben wurde jedoch auch eine intrinsische Aktivität, i.S. einer am Benzodiazepin-Rezeptor agonistischen oder invers agonistischen Aktivität beschrieben.

\section{Abb. 2 Veränderungen der Konzentration neuroaktiver Steroide}
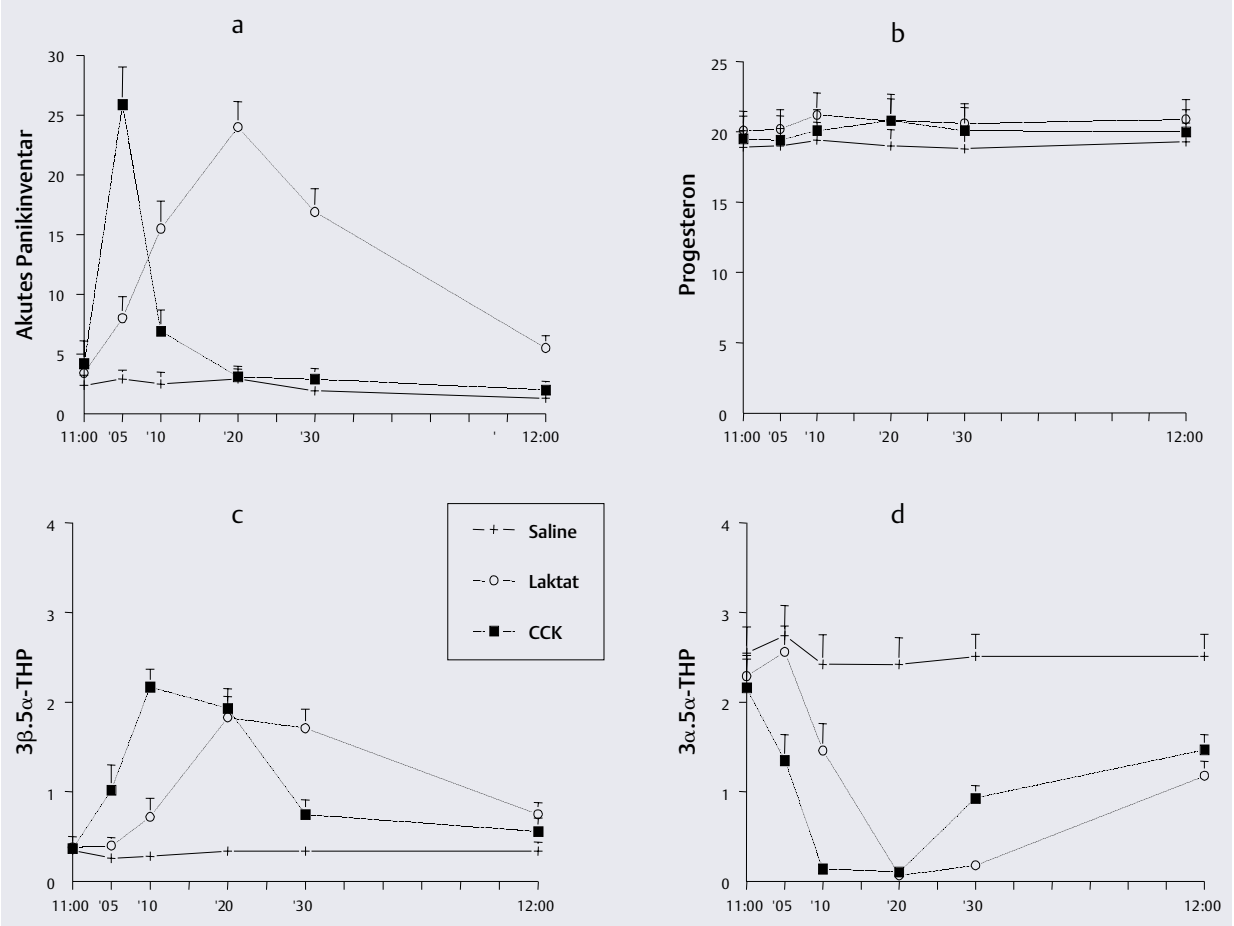

Mittlerer ( \pm SEM) API-Score (a), Progesteron (b), 3 $\beta, 5 \alpha-$ THP (c) und $3 \alpha, 5 \alpha-T H P$ (d) Konzentrationen unter Plazebo (Saline), Laktat und CCK-4
Die 1990 von Nutt und Mitarbeitern (10) postulierte „RezeptorShift“ bzw. „Set-Point“ Hypothese der Panikstörung basiert auf der Beobachtung, dass acht der zehn untersuchten Patienten mit einer Panikstörung eine Panikattacke nach Flumazenil entwickelten. Bei gesunden Kontrollprobanden fanden sich keine Hinweise auf eine anxiogene Aktivität von Flumazenil. Dieser Befund veranlasste uns, die Effekte von Flumazenil bei Patienten mit Panikstörung mit der am besten untersuchten panikogenen Substanz, einer Laktat-Lösung, zu vergleichen. Während acht der zehn untersuchten Patienten eine Laktatinduzierte Panikattacke entwickelten, konnte durch Flumazenil-Gabe keine Panikattacke experimentell induziert werden (19). Neben den fehlenden psychopathologischen Veränderungen fand sich nach Flumazenil auch keine Aktivierung des HPA-Systems oder der untersuchten Kreislaufparameter, so dass sich insgesamt keine Hinweise für eine invers agonistische Aktivität von Flumazenil bei den untersuchten Patienten mit Panikstörung ergaben. Auch im Vergleich zu gesunden Kontrollprobanden fand sich bei den untersuchten Patienten mit einer Panikstörung keine differentielle Wirksamkeit von Flumazenil i.S. einer invers agonistischen Aktivität von Flumazenil oder einen „Rezeptor-Shift“ bzw. eine veränderte Rezeptoreinstellung („Set-Point“). Möglicherweise ist eine Kombination aus komorbiden psychiatrischen Erkrankungen, einer hohen Panikattacken-Frequenz und Effekten des weiblichen Zyklus an der panikogenen Aktivität von Flumazenil mit beteiligt. Unsere Ergebnisse werden auch von Randall und Mitarbeitern (13) gestützt, die ebenfalls keine Hinweise auf eine panikogene Aktivität von Flumazenil bei Patienten mit PTSD und komorbider Panikstörung fanden.

In den letzten Jahren fanden sich vermehrt Hinweise dafür, dass bestimmte Steroide durch Interaktion mit Neurotransmitter-Rezeptoren auch die neuronale Erregbarkeit über membranäre Prozesse modulieren können. Steroide mit diesen Eigen- 
Abb. 3 Modulation des Stresshormonsystems durch ANP

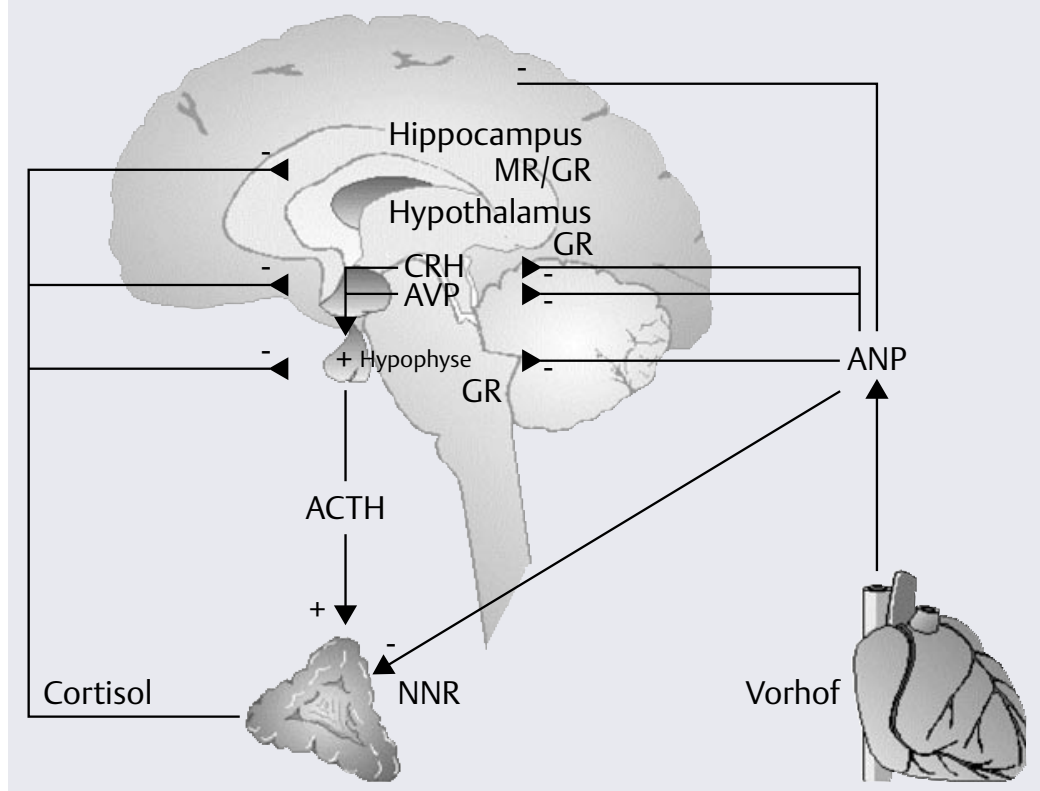

Abkürzungen: AVP Arginin Vasopressin; ANP Atriales natriuretisches Peptid; CRH Corticotropin Releasing Hormon; HPA-System Hypothalamic Pituitary Adrenocortical System; GR Glukokortikoid-Rezeptor; MR Mineralokortikoid-Rezeptor; NNR Nebennierenrinde

schaften werden als neuroaktive Steroide bezeichnet und können teilweise im Gehirn ohne Zuhilfenahme peripherer Ressourcen synthetisiert werden. Die beiden neuroaktiven Steroide $3 \alpha, 5 \alpha$-THP und $3 \alpha, 5 \alpha$-THDOC modulieren die neuronale Erregbarkeit durch Interaktion mit den $\mathrm{GABA}_{\mathrm{A}}$-Rezeptoren. Diese neuroaktiven Steroide zeigen in elektrophysiologischen Untersuchungen Benzodiazepin-ähnliche Effekte und sind entsprechend auch anxiolytisch und hypnotisch wirksam. Andere neuroaktive Steroide haben gegensinnige Wirkungen. Bei experimentell induzierten Panikattacken von Patienten mit Panikstörung kommt es nahezu zeitgleich zu den psychopathologischen Veränderungen zu massiven Veränderungen der Konzentration neuroaktiver Steroide, die von einem stark verminderten GABA-ergen Tonus begleitet sein können (Abb. 2) (23). Bemerkenswerterweise fanden sich diese Veränderungen nicht bei Kontrollprobanden, nicht einmal bei den Kontrollprobanden mit experimentell induzierten Panikattacken. Basal zeigten die Patienten mit Panikstörung erhöhte Konzentrationen der $\mathrm{GABA}_{\mathrm{A}}$-agonistisch wirksamen Stereoisomere $3 \alpha, 5 \alpha$-THP und $3 \alpha, 5 \beta$ THP, während das $\mathrm{GABA}_{\mathrm{A}}$-antagonis- tisch wirksame Stereoisomer $3 \beta, 5 \alpha-$ THP vermindert war. Die Panikattacken-assoziierten Veränderungen neuroaktiver Steroide lassen es möglich erscheinen, dass die basalen Veränderungen der Konzentrationen neuroaktiver Steroide bei Patienten mit einer Panikstörung kompensatorisch erhöht sind, um dem Auftreten von Panikattacken entgegenzuwirken.

\section{- Möglichkeiten und Grenzen}

Allgemeine Ziele, die mit der experimentellen Symptomprovokation verfolgt werden können, sind in Tabelle 2 aufgeführt. Neben den dargestellten Untersuchungen zur Pathophysiologie und Therapie der Panikstörung werden z.B. $\mathrm{CO}_{2}$-induzierte Panikattacken auch für genetische Studien herangezogen. Während die wissenschaftliche Untersuchung experimentell induzierter Panikattacken weltweit an vielen Zentren durchgeführt wird, hat sich die vorgeschlagene Anwendung zur Behandlung der Panikstörung (1) bisher nicht durchgesetzt. Neben praktischen Aspekten ist hierfür insbesondere die gute Behandlungsmöglichkeit der Panikstörung verantwortlich.

Die Behandlungssensitivität eines experimentelles Modells ist ein wichtiger Aspekt der Validität. Während pharmakologische Interventionen, wie in Tabelle 3 dargestellt, gut untersucht sind, gibt es zum Einfluss einer kognitiven Verhaltenstherapie bisher nur eine kleine Studie von Shear et al. (16), die eine Abschwächung laktatinduzierter Panikattacken bei Patienten beschreiben konnte.

Da Laktat auch bei gesunden Probanden mit Panikattacken in der Vorgeschichte oder bei Patienten mit Panikattacken i.R. anderer psychiatrischer Erkrankungen panikogen ist, ist die Spezifität nicht auf die Panikstörung, sondern auf die Panikattacke als klinisches Syndrom begrenzt. CCK-4 dahingegen ist dosisabhängig auch bei gesunden Kontrollprobanden anxiogen bzw. panikogen.

\section{Mögliche neue Therapieansätze}

Am Beispiel eigener Arbeiten mit dem atrialen natriuretischen Peptid (ANP) wird dargestellt, dass die experimentelle Provokation von Panikattacken verwendet werden kann, um mögliche neue Behandlungsansätze zu untersuchen. Allerdings hat sich in Studien zur therapeutischen Anwendung von CCK-BRezeptor-Antagonisten gezeigt, dass die anxiolytische Aktivität bei experimentell induzierten Panikattacken nicht gleichzusetzen ist mit der klinischen Wirksamkeit bei Patienten. Auch neue Antiepileptika werden derzeit unter Anwendung experimentell induzierter Panikattacken als mögliche neue Behandlungsansätze untersucht (25). Inwiefern experimentelle Panikattacken von Patienten mit Panikstörung und gesunden Kontrollprobanden vergleichbar sind, wurde bisher nicht systematisch untersucht. Es gibt jedoch Hinweise aus eigenen Untersuchungen, dass Panikattacken von Patienten und Probanden auch neurobiologisch verschieden sein können (23).

\section{Atriales Natriuretisches Peptid (ANP)}

Das atriale natriuretische Peptid (ANP) besteht aus 28 Aminosäuren, und wird v.a. im rechten Vorhof des Herzens synthetisiert (5). Neben 


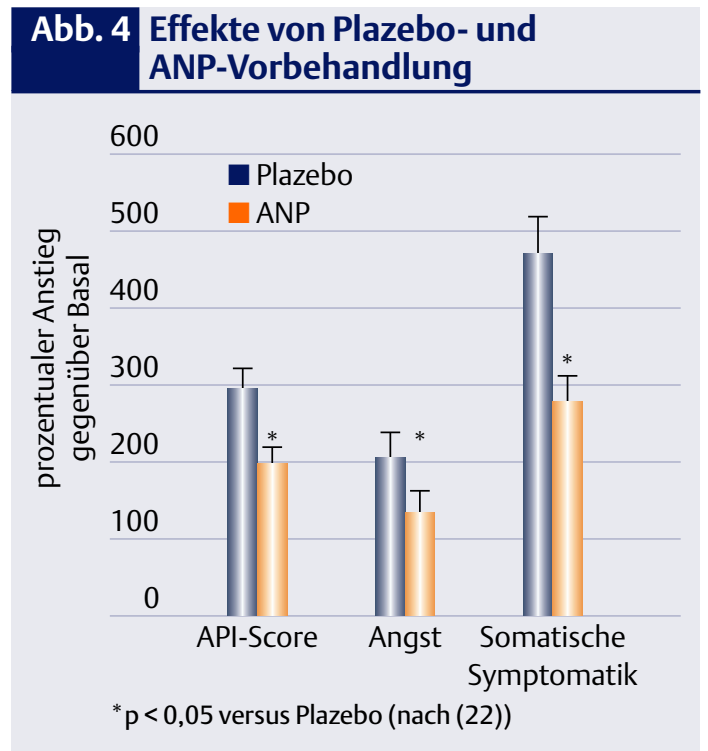

Normierter CCK-4-induzierter Anstieg des APIScores und des Angst- und Somatischen Subscores

ANP wurden zwei verwandte Peptidhormone beschrieben: Brain Natriuretic Peptid (BNP) und C-type natriuretic Peptid (CNP). BNP wurde im Gehirn des Schweins entdeckt, beim Menschen jedoch wird es hauptsächlich in den Ventrikeln des Herzens synthetisiert und hat primär hämodynamische Funktionen. ANP und CNP sind im ZNS weit verbreitet und spielen eine wichtige Rolle als Neuromodulator.

In-vivo- und in-vitro-Studien bei der Ratte konnten zeigen, dass ANP als ein hypothalamischer CRH-inhibierender Faktor wirkt. Die intravenöse Gabe von ANP-Antiserum hatte bei der Ratte einen deutlichen Anstieg der basalen wie auch der stimulierten HPA-Aktivität zur Folge. In-vivo-Studien beschrieben auch einen direkt hemmenden Effekt von ANP auf die adrenale Kortisolfreisetzung. Auch beim Menschen konnte gezeigt werden, dass ANP die CRHstimulierte ACTH-, Kortisol- und Prolaktinsekretion hemmt (20). Die Interaktion von ANP mit dem Stresshormonsystem ist in Abbildung 3 dargestellt.

Während die neuroendokrinologische Funktion und die Rolle natriuretischer Peptide in der Volumenregulation gut charakterisiert sind, wurden hiervon unabhängige Verhaltenseffekte natriuretischer Peptide bisher kaum untersucht. Erste
Hinweise für eine Verhaltensaktivität von ANP kamen aus Untersuchungen, die ein verändertes Explorationsverhalten von Ratten im „Open-Field-Test" sowie eine verzögerte Extinktion angstassoziierten Verhaltens beschrieben. Erste Hinweise für eine mögliche Rolle von ANP bei Patienten mit einer Panikstörung ergaben sich aus einer Studie von Kellner et al. (9), in der gezeigt werden konnte, dass Laktat-induzierte Panikattacken von Patienten mit einer Panikstörung von einer vermehrten ANP-Freisetzung begleitet sind. Diese vermehrte ANP-Freisetzung erklärt möglicherweise die fehlende Aktivierung des HPA-Systems bei Laktat-induzierten Panikattacken. In den nachfolgenden Untersuchungen wird die mögliche Rolle von ANP in der Angstmodulation beschrieben. Dies wurde sowohl präklinisch im Tierexperiment, als auch klinisch bei Patienten mit einer Panikstörung untersucht.

Im Tierexperiment konnte eine anxiolytische Aktivität von Atriopeptin II, einem ANP-Fragment, beschrieben werden. Sowohl nach periphererer als auch nach zentraler Gabe von Atriopeptin II fand sich im Elevated Plus-Maze nach Social Defeat eine vermehrte Zeit auf dem sowie vermehrt Eintritte in den offenen Arm des Plus Maze, was als verminderte Angst interpretiert wird. Eine vergleichbare anxiolytische Aktivität zeigten die Tiere nach lokaler Applikation von Atriopeptin II in den zentralen Amygdalakern (18). Die spezifische anxiolytische Aktivität von ANP bei der Ratte wird durch die fehlenden Effekte auf die lokomotorische Aktivität im Open Field-Test unterstrichen, denn eine unveränderte lokomotorische Aktivität ist Voraussetzung für die korrekte Interpretation der Verhaltenstests.

Auch bei Patienten mit einer Panikstörung konnte eine anxiolytische Aktivität von ANP beschrieben werden: die CCK-4-induzierte Symptomatik von Patienten wurde durch Vorbehandlung mit ANP signifikant abgeschwächt (22). Neben den CCK-4-induzierten somatischen Symptomen waren auch angstspezifische Variablen des API-Scores durch ANP-Vorbehandlung signifikant abgeschwächt (s. Abb. 4). Aufgrund der kleinen Stichprobe fand sich jedoch nur ein Trend zu einer verminderten Anzahl CCK-4-induzierter Panikattacken nach ANP-Vorbehandlung. Eine anxiolytische Aktivität von ANP beim Menschen wurde auch in einer Studie von Wiedemann und Mitarbeitern (24) nachgewiesen. Darüber hinaus konnte gezeigt werden, dass die anxiolytische Aktivität von ANP bei Patienten mit Panikstörung und gesunden Kontrollprobanden von einer Hemmung des HPA-Systems und einer Beeinflussung der Herzfrequenzvariabilität begleitet war (24). In dieser Studie wurde eine Dosis von CCK-4 verabreicht, die auch bei einem Großteil der Kontrollprobanden eine Panikattacken-ähnliche Symptomatik hervorrief. Dies deutet darauf hin, dass die anxiolytische Aktivität von ANP beim Menschen möglicherweise nicht nur auf Patienten mit einer Panikstörung beschränkt ist.

Sowohl tier- als auch humanexperimentelle Studien beschreiben also eine anxiolytische Aktivität von ANP, so dass sich die Frage stellt, inwiefern eine Modulation der Rezeptoren natriuretischer Peptide einen neuen Therapieansatz in der Behandlung von Angsterkrankungen, z.B. auch der Panikstörung, darstellen könnte. Da es sich bei spezifischen Angststörungen und insbesondere auch der Panikstörung um chronisch verlaufende psychiatrische Störungen handelt, müssen Therapien auch zur langfristigen Anwendung geeignet sein. Die parenterale Gabe stellt nur ein Mittel zur wissenschaftlichen Untersuchung von Peptiden dar, da sie therapeutisch zur Behandlung chronisch verlaufender Angsterkrankungen nicht genutzt werden kann. Derzeit limitiert also noch die fehlende Verfügbarkeit nichtpeptiderger ANP-Rezeptorliganden mit oraler Bioverfügbarkeit die weitere Untersuchung von ANP in der Therapie der Panikstörung.

\section{Schlussfolgerungen}

Neben primär pathophysiologisch orientierten wissenschaftlichen Untersuchungen kann die ex- 
perimentelle Provokation von Panikattacken genutzt werden, um mögliche neue Therapieansätze zu untersuchen. Dies wurde am Beispiel des atrialen natriuretischen Peptids dargestellt. Inwiefern hieraus abgeleitete Therapiestrategien in die Klinik Eingang finden werden, wird sich in den kommenden Jahren zeigen. Obwohl die Neurobiologie von Angsterkrankungen in vielen Bereichen noch unklar ist, stellen experimentelle Panikattacken eine Möglichkeit dar, ein so komplexes Phänomen wie Angst standardisiert zu untersuchen und neue Behandlungsstrategien zu entwickeln.

\section{Experimentally induced panic attacks}

The diagnostic symptom provocation has a long tradition in medicine. In psychiatry, symptom provocation studies are used to study the pathophysiology and treatment of disorders. Suddenly, and unexpected panic attacks have a characteristic course and a typical pattern of somatic, cognitive, emotional and behavioral symptoms. Beginning with the study of Pitts and McClure who described the panicogenic activity of sodium lactate, the experimental induction of panic attacks with different challenges has been used to characterize the neurobiology of anxiety. Furthermore, experimentally induced panic attacks can be used to study possible new treatment approaches. An anxiolytic activity of atrial natriuretic peptide suggests that modulation of natriuretic peptide receptors with nonpeptidergic ligands may be a new treatment approach. Experimentally induced panic attacks are a tool to characterize the neurobiology of anxiety and panic and may be used to develop new treatment approaches.

\section{Key Words}

panic disorder - panic - anxiety experimental anxiety - neurobiology of anxiety

\section{Literatur}

1. Bonn JA, Harrison J, Rees L. Lactate infusion in the treatment of 'free-floating' anxiety. Canad Psychiat Ass J 1973; 18: 41-46 2. Bradwejn J, Koszycki D, Shriqui C. Enhanced sensitivity to cholecystokinin tetra- petide in panic disorder. Arch Gen Psychiatry 1991; 48: 603-610

3. Bradwejn J, Koszycki D. Imipramine antagonism of the panicogenic effects of cholecystokinin tetrapeptide in panic disorder patients. Am J Psychiatry 1994; 151: 261-263

4. Charney DS, Heninger GR, Breier A. Noradrenergic function in panic anxiety. Effects of yohimbine in healthy subjects and patients with agoraphobia and panic disorder. Arch Gen Psychiatry 1984; 41: 751-763

5. De Bold AJ, Borenstein HB, Veress AT, Sonnenberg $\mathrm{H}$. A rapid and potent natriuretic response to intravenous injection of atrial myocardial extracts in rats. Life Sci 1981; 28: 89-94

6. Dillon DJ, Gorman JM, Liebowitz MR, Fyer AJ, Klein DF. Measurement of lactate-induced panic and anxiety. Psychiatry Res 1987; 20: 97-105

7. Gorman JM, Fyer MR, Goetz R, Askanazi J, Liebowitz MR, Fyer AJ, Kinney J, Klein DF. Ventilatory physiology of patients with panic disorder. Arch Gen Psychiatry 1988; 45 : 31-39

8. Kahn RS, Asnis GM, Wetzler S, van Praag HM. Neuroendocrine evidence for serotonin receptor hypersensitivity in panic disorder. Psychopharmacology (Berl) 1988; 96: 360-364

9. Kellner M, Herzog L, Yassouridis A, Holsboer $\mathrm{F}$, Wiedemann $\mathrm{K}$. A possible role of atrial natriuretic hormone in pituitaryadrenocortical unresponsiveness in lactateinduced panic. Am J Psychiatry 1995; 152: 1365-1367

10. Nutt DJ, Glue P, Lawson C, Wilson S. Flumazenil provocation of panic attacks. Evidence for altered benzodiazepine receptor sensitivity in panic disorder. Arch Gen Psychiatry 1990; 47: 917-925

11. Perna G, Bertani A, Caldirola D, Bellodi L. Family history of panic disorder and hypersensitivity to $\mathrm{CO} 2$ in patients with panic disorder. Am J Psychiatry 1996; 153: 1060-1064

12. Pitts FM, McClure JN. Lactate metabolism in anxiety neurosis. New England J Med 1967; 177: 1329-1336

13. Randall PK, Bremner JD, Krystal JH, Nagy LM, Heninger GR, Nicolaou AL, Charney DS. Effects of the benzodiazepine antagonist flumazenil in PTSD. Biol Psychiatry 1995; 138: 319-324

14. Roy-Byrne PP, Cowley DS, Greenblatt DJ, Shader RI, Hommer D. Reduced benzodiazepine sensitivity in panic disorder. Arch Gen Psychiatry 1990; 47: 534-538

15. Seibyl JP, Krystal JH, Price LH, Woods SW, D'Amico C, Heninger GR, Charney DS. Effects of ritanserin on the behavioral, neuroendocrine, and cardiovascular responses to meta-chlorophenylpiperazine in healthy human subjects. Psychiatry Res 1991; 38: 227-236

16. Shear MK, Fyer AJ, Ball G, Josephson S, Fitzpatrick M, Gitlin B, Frances A, Gorman J, Liebowitz M, Klein DF. Vulnerability to sodium lactate in panic disorder patients given cognitive behavioral therapy. Am J Psychiatry 1991; 148: 795-797

17. Shlik J, Aluoja A, Vasar V, Vasar E, Podar
T, Bradwejn J. Effects of citalopram treatment on behavioral cardiovascular and neuroendocrine response to cholecystokinin tetrapeptide challenge in patients with panic disorder. J Psychiatry Neurosci 1997; 22: 332-340

18. Ströhle $A$, Jahn $H$, Montkowski $A$ Liebsch G, Boll E, Landgraf R, Holsboer F, Wiedemann K. Central and peripheral administration of atriopeptin is anxiolytic in rats. Neuroendocrinology 1997; 65: 210-215

19. Ströhle A, Kellner M, Yassouridis A, Holsboer F, Wiedemann K. Effect of flumazenil in lactate-sensitive patients with panic disorder. Am J Psychiatry 1998; 155: 610-612

20. Ströhle A, Kellner M, Holsboer F, Wiedemann K. Atrial natriuretic hormone decreases endocrine response to a combined dexamethasone corticotropin-releasing hormone test. Biol Psychiatry 1998; 43: 371-375

21. Ströhle A, Holsboer F, Rupprecht R. Increased ACTH concentrations associated with cholecystokinin tetrapeptide-induced panic attacks in patients with panic disorder. Neuropsychopharmacology 2000; 22: $251-256$

22. Ströhle A, Kellner M, Holsboer F, Wiedemann K. Anxiolytic activity of atrial natriuretic peptide in patients with panic disorder. Am J Psychiatry 2001; 158: 1514-1516

23. Ströhle A, Romeo E, di Michele F, Pasini A, Holsboer F, Rupprecht R. Induced panic attacks shift GABAA receptor modulatory neuroactive steroid composition. Preliminary results. Arch Gen Psychiatry 2003; 60: 161-168

24. Wiedemann $\mathrm{K}$, Jahn $\mathrm{H}$, Yassouridis $\mathrm{A}$, Kellner M. Anxiolytic activity of atrial natriuretic peptide on cholecystokinin tetrapeptide-induced panic attacks. Arch Gen Psychiatry2001; 58: 371-377

25. Zwansger P, Baghai TC, Schuele C, Ströhle A, Padberg F, Kathmann N, Schwarz $\mathrm{M}$, Möller H-J, Rupprecht R. Vigabatrin decreases cholecystokinin-tetrapeptide (CCK-4) induced panic in healthy volunteers. Neuropsychopharmacology 2001; 25: 699-703

\section{Korrespondenzadresse:}

Priv.-Doz. Dr. med. Andreas Ströhle

Klinik für Psychiatrie und Psychotherapie

Charité Campus Mitte

Charité - Universitätsmedizin Berlin

Schumannstr. 20/21

10117 Berlin

andreas.stroehle@charite.de 